\title{
GDC seeks views on 'working with the dental team for public safety and confidence'
}

The GDC has launched a consultation on its strategy for 2020-22 'Working with the dental team for public safety and confidence.' The consultation seeks, for the first time, a conversation about the choices to be made in delivering the regulator's broad statutory objectives.

The strategy reflects the significant progress the GDC has made against its ambitions to make dental regulation better and fairer - as described in Shifting the balance - and the many difficult decisions taken to reduce costs and improve effectiveness.

According to the document, the strategy sets out a vision for the GDC to:

$\rightarrow$ Protect patients while being fair to registrants, cost-effective and proportionate: by focusing on education and engagement, supporting career-long learning and promoting high standards of care and professional conduct

$\rightarrow$ Work with the professions and partners to ensure that public concerns can be addressed by the right body, quickly and effectively

$\rightarrow$ Use evidence, research and evaluation to ensure enforcement is cost-effective and right-touch

$\rightarrow$ Ensure its approach to regulation keeps pace with change

$\rightarrow$ Continue to build on the considerable improvements it has made.

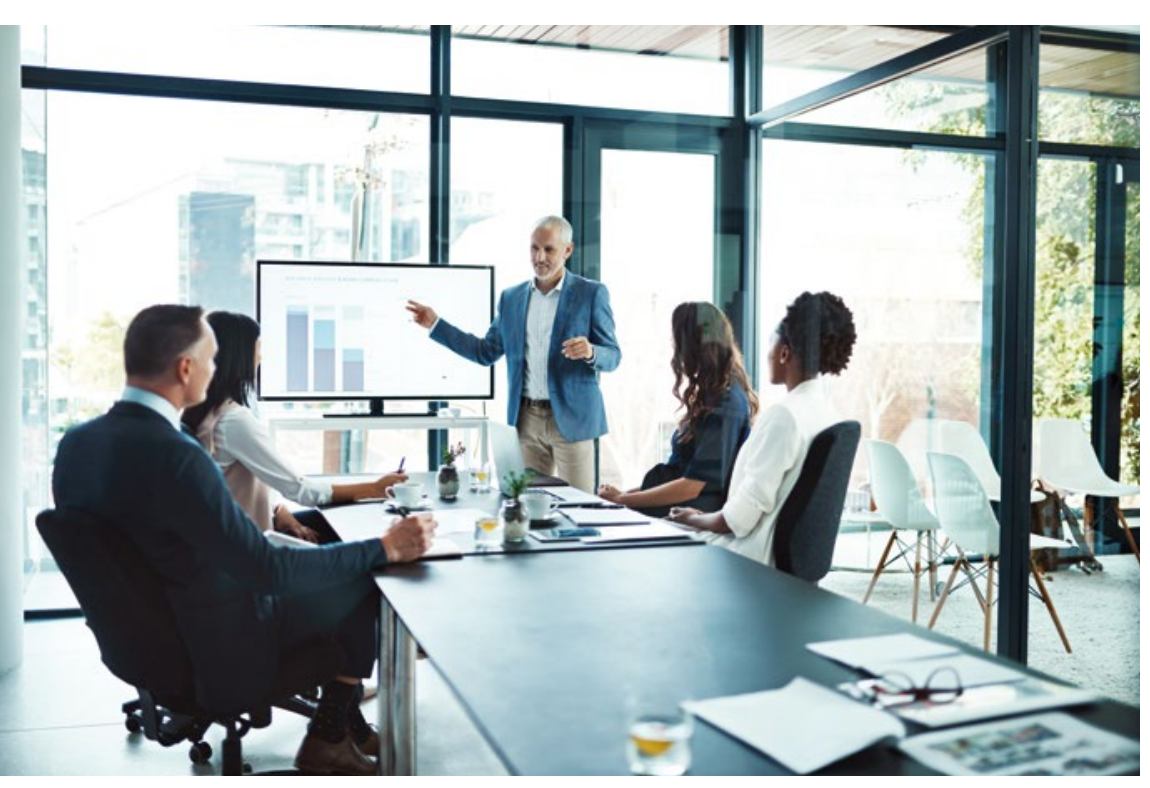

Whilst the GDC is clear that this is not a consultation on fee levels, it recognises that those levels are fundamentally affected by the costs of the Strategy and addresses the ARF accordingly. Because of the significant achievements that have been made in improving financial control and reducing costs, the GDC is expecting the strategy to be consistent with a significant reduction in the ARF for dentists and only a very small increase in the fee level for dental care professionals.

GDC Chief Executive and Registrar, Ian Brack, said: 'The GDC is committed to changing the regulatory landscape, for the reasons we identified in Shifting the balance. The priorities we identified in that document remain at the core of our ambition for the next three years. We have come a long way toward our goal of achieving a better, fairer system of dental regulation, but we have a great deal more to do and this strategy sets out our objectives for the next three years. This consultation provides an opportunity for people to have their say on those objectives as well as the priority and resourcing we afford to each. I hope that opportunity is taken up and as many people as possible give their views.'

The consultation is open now and can be accessed on the GDC's website. The deadline to respond is Thursday 30 July 2019. 